\title{
Editorial
}

\section{El mundo de la ciencia y la tecnología de los materiales}

Gabriel Peña Rodríguez ${ }^{1}$

$\mathrm{L}$ a evolución del ser humano y por ende de la sociedad, ha estado marcada por la capacidad de producir sus herramientas o materiales necesarios para satisfacer las necesidades básicas. Lo anterior, ha permitido históricamente la clasificación de las civilizaciones según los materiales utilizados, por ejemplo la edad de piedra, del cobre, del bronce y la del hierro. En cada una de estas edades ha sido necesaria la construcción de tecnologías, que permitan una mejor producción de los materiales usados, siendo cada vez más complejas, a medida que se conocían los nuevos materiales. Inicialmente se utilizaron los materiales naturales como la piedra, madera, pieles, arcilla entre otros, posteriormente, y a medida que se descubrían nuevas técnicas, se usaron otros materiales con propiedades superiores a los llamados naturales.

A medida que el hombre ha podido conocer, la relación entre los elementos constituyentes de los materiales y sus propiedades, ha permitido su clasificación, y por ende su aplicación tanto a nivel doméstico como industrial. En la actualidad, se desarrollan gran variedad de materiales con propiedades muy especiales para satisfacer las necesidades de la sociedad, donde se han utilizado metales, cerámicos, plásticos, vidrios, fibras y los llamados materiales compuestos o composites.

El estudio o análisis de la relación entre la estructura y las propiedades de los materiales, es lo que se conoce como la ciencia de los materiales, mientras que la ingeniería de materiales se fundamenta en las relaciones entre estructura-procesamiento-funcionamiento, para diseñar y producir materiales con propiedades predeterminadas. La ciencia de materiales es una disciplina multidisciplinaria donde incluye elementos de la química, biología, matemática y física, así como de ingenieras mecánica, civil, eléctrica, electrónica, entre otras, para el estudio de las propiedades macroscópicas y estructurales de los materiales, que puedan ser utilizados en maquinas y herramientas, o en productos requeridos a nivel industrial.

Con la aparición de nuevas tecnologías o instrumentos (SEM, DRX, FRX, TEM, FTIR, DSC, TGA, entre otros), cada vez más sofisticados, han permitido conocer el mundo micro-meso y nanoscopico, adentrando a la sociedad en la época de los polímeros, semiconductores, materiales compuestos y nuevos materiales, incursionándose en lo que hoy se llama la nanociencia y nanotecnología.

Siendo más específicos, por ejemplo en la industria de la micro y optoelectrónica, se habla de la "era del silicio", la cual a partir del descubrimiento del "grafeno", por los investigadores Andre Geim y Konstantin Novoselov, descubrimiento que les permitió ser los ganadores del premio Nobel en Física 2010, muy probablemente será reemplazada por la "era del grafeno", material que es una alotropía del carbono, caracterizado por tener una alta conductividad térmica y eléctrica, por combinar elasticidad y ligereza con dureza. Dentro de las aplicaciones de este material, se habla de la construcción de pantallas para televisores y computadores flexibles (enrollables); de dispositivos para la industria electrónica con velocidades de procesamiento del orden de los cientos de giga-bytes y auto-refrigeradas; de uso en el blindaje de carros y construcción de chalecos anti balas, debido a su dureza la cual es mayor que la del diamante y unos cientos de veces mayor a la del acero; en células solares, debido a su alta conductividad eléctrica; en sensores de gas de estado sólido y en otras mucha más, que en la actualidad son investigadas en las universidades y centros de investigación de todo el mundo.

Por todo lo anterior, es de vital importancia que en las universidades del país se sigan creando programas de posgrado a nivel de maestría y doctorado, en ciencia y tecnología de materiales, los cuales junto con la actualización y adquisición de equipos propios, permitan realizar investigaciones que generen indicadores de propiedad intelectual para los investigadores, las instituciones y el País.

${ }^{1}$ Doctor en ingeniería de materiales.

Profesor asociado adscrito al departamento de Física-UFPS 